INPLASY

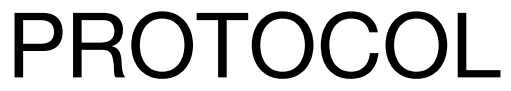

To cite: Wang et al. Efficacy and safety evaluation of hyperbaric oxygen therapy for patients with ulcerative colitis: A protocol of systematic review and meta-analysis. Inplasy protocol 2020100118. doi:

10.37766/inplasy2020.10.0118

Received: 30 October 2020

Published: 31 October 2020

Corresponding author:

Xiao-dong Zhao

13810490288@163.com

Author Affiliation:

Dongzhimen Hospital, Beijing University of Chinese Medicine, Beijing, China

Support: National Natural Science.

Review Stage at time of this submission: The review has not yet started.

Conflicts of interest:

The authors have no conflicts of interest to disclose.

\section{Efficacy and safety evaluation of hyperbaric oxygen therapy for patients with ulcerative colitis: A protocol of systematic review and meta-analysis}

Wang, W1; He, Y2; Wen, D3; Zhao, XD4; Jiang, SS5.

Review question / Objective: This review aims to systematically investigate the efficacy and safety of hyperbaric oxygen therapy (HBOT) for ulcerative colitis (UC) patients reported in randomized clinical trials (RCTs).

Condition being studied: UC is a chronic inflammatory disease affecting the colon whose pathogenesis is multifactorial, involving a genetic predisposition, epithelial barrier defects, dysregulated immune responses, and environmental factors. And it usually presents with bloody diarrhoea and is diagnosed by colonoscopy and histological findings. The incidence of UC is rising worldwide, most commonly afflicting adults aged 30-40 years and resulting in disability. And it is becoming one of the clinically refractory diseases.

INPLASY registration number: This protocol was registered with the International Platform of Registered Systematic Review and Meta-Analysis Protocols (INPLASY) on 31 October 2020 and was last updated on 31 October 2020 (registration number INPLASY2020100118).

\section{INTRODUCTION}

Review question / Objective: This review aims to systematically investigate the efficacy and safety of hyperbaric oxygen therapy (HBOT) for ulcerative colitis (UC) patients reported in randomized clinical trials (RCTs).

Condition being studied: UC is a chronic inflammatory disease affecting the colon whose pathogenesis is multifactorial, 
involving a genetic predisposition, epithelial barrier defects, dysregulated immune responses, and environmental factors. And it usually presents with bloody diarrhoea and is diagnosed by colonoscopy and histological findings. The incidence of UC is rising worldwide, most commonly afflicting adults aged $30-40$ years and resulting in disability. And it is becoming one of the clinically refractory diseases.

\section{METHODS}

Search strategy: Search medical subject headings (MeSH) and key words associated with HBOT in the treatment of UC based on the following databases: Embase, MEDLINE, PubMed, Web of Science, Cochrane Library Central Register of Controlled Trials, the Chinese Biomedical Literature Database (CBM), China national knowledge infrastructure database (CNKI), Wan fang database, Chongqing VIP information, Google scholar, Baidu Scholar, and SinoMed.

Participant or population: Patients who are clinically diagnosed with UC will be included, regardless of their gender, age, ethnicity, severity of the disease, economic status, or education.

Intervention: The intervention measures of the treatment group were HBOT alone or as a combination with routine treatment recommended by guidelines.

Comparator: The intervention measures of the control group will include no treatment, placebo, and conventional pharmacotherapies recommended by guidelines.

Study designs to be included: RCTs that investigated the efficacy and safety of HBOT for UC patients will be included.

Eligibility criteria: The study will only select RCTs. Non-randomized controlled trials, reviews, case reports, experimental studies, expert experience and duplicate publications will be excluded. Meanwhile, only participants who are clinically diagnosed with UC will be included, and some special participants who are pregnant or nursing women or with severe heart, liver or lung disease will be excluded.

Information sources: The database of randomized controlled trials related to HBOT of UC was reviewed to collect the following: Embase, MEDLINE, PubMed, Web of Science, Cochrane Library Central Register of Controlled Trials, CBM, CNKI, Wan fang database, Chongqing VIP information, Google scholar, Baidu Scholar, and SinoMed. No limitation on language or publication types restriction will be applied.

Main outcome(s): Mainly observe the clinical effectiveness.

Quality assessment / Risk of bias analysis: Two authors will independently assess the risk of bias on the basis of the Cochrane risk-of-bias tool for each study included. In addition, a third party will be invited to resolve disagreement.

Strategy of data synthesis: The software RevMan 5.3 will be used to perform the meta-analysis. If heterogeneity is minor, a fixed-effect model will be used for metaanalysis. However, if heterogeneity is substantial, a random-effects model will be used for meta-analysis. Moreover, we will carry out subgroup analysis or other assessments to check the possible reasons.

Subgroup analysis: If necessary, we will perform subgroup analysis to study heterogeneity according to interventions, participant characteristics, and outcome measures.

Sensibility analysis: Sensitivity analysis will be used to assess the robustness of the results.

Language: No language restrictions.

Country(ies) involved: China.

Keywords: hyperbaric oxygen therapy, ulcerative colitis, systematic review, protocol. 
Contributions of each author:

Author 1 - Wei Wang.

Author 2 - Ying He.

Author 3 - Dou Wen.

Author 4 - Xiao-dong Zhao.

Author 5 - Shang-shang Jiang. 